Available online at www.eccomasproceedia.org

Eccomas Proceedia UNCECOMP (2021) 313-322

ECCOMAS

Proceedia
UNCECOMP 2021

$4^{\text {th }}$ ECCOMAS Thematic Conference on Uncertainty Quantification in Computational Sciences and Engineering M. Papadrakakis, V. Papadopoulos, G. Stefanou (eds.) Streamed from Athens, Greece, 28 -30 June 2021

\title{
EFFECTIVENESS OF THE PROBABILITY DENSITY EVOLUTION METHOD FOR DYNAMIC AND RELIABILITY ANALYSES OF MASONRY STRUCTURES
}

\author{
Massimiliano Lucchesi ${ }^{1}$, Barbara L. Pintucchi ${ }^{1}$, and Nicola Zani ${ }^{1}$ \\ ${ }^{1}$ University of Florence, Department of Civil and Environmental Engineering \\ via S. Marta 3, Florence, Italy \\ e-mail: \{massimiliano.lucchesi, barbara.pintucchi, nicola.zani\}@unifi.it
}

\begin{abstract}
The main objective of this paper is to examine the possibility of using the probability density evolution theory (PDEM) to determine the evolution of the probability of some structural parameters, during dynamic processes of masonry buildings subjected to seismic actions. The study is mainly motivated by the computational burden that is required by the Monte Carlo method in the case of step-by-step dynamic analyses of structures with large size, complex geometry and a highly non-linear constitutive equation. The PDEM requires the deterministic solution of the dynamic system in a limited number of cases (much lower than that required by the Monte Carlo method), together with the numerical solution of a linear partial differential equation of the first order. First of all, the effectiveness of the method is verified in the case of a simple problem whose explicit solution is known, mainly to determine the most suitable numerical method for solving the differential equation. Then, the dynamic behavior of a masonry tower is analysed. The structure is modeled as a beam with a hollow rectangular section, made of a no-tension material with softening in compression. It is subjected to the action of a real earthquake. The Young's modulus of the material is assumed to be a random variable, and the probability density function of the displacement at the top of the tower is determined throughout the time-history. The results obtained at some time-steps are compared with those provided by the Monte Carlo method. Although the example examined is quite simple, the PDEM appears to be very promising to study more complex masonry structures.
\end{abstract}

Keywords: Masonry, Tower, Structural dynamics, Mechanical parameters uncertainties, Probability Density Evolution Method.

ISSN:2623-3339 (C) 2021 The Authors. Published by Eccomas Proceedia.

Peer-review under responsibility of the organizing committee of UNCECOMP 2021.

doi: $10.7712 / 120221.8040 .19025$ 


\section{INTRODUCTION}

The analyses of structures subjected to dynamic loads are now generally conducted using refined mechanical models and adequate numerical techniques. However, even when the problem is well posed so that both the existence and the uniqueness of the solution are guaranteed, the parameters describing the geometric and mechanical characteristics of the structures and the external actions are generally affected by uncertainties that must be taken into account.

In general, the state equations of the dynamic system - typically obtained by discretizing a structure into finite elements, and the initial joint probability density function (Pdf) of all the considered random variables are assigned. Thus, a stochastic process is obtained that is parametrized over the time, and has the Euclidean space as state space.

In many applications it is necessary to address the problem of determining the Pdf of some quantities of interest at predetermined times. This goal is in most cases achieved using the Monte Carlo method which, at least in principle, allows to consider complex models and geometries without having to resort to unrealistic simplifying hypotheses. On the other hand, this method can require extremely long computational times [1]. For this reason, the probabilistic analyses of masonry structures are rarely conducted via dynamic (time-history) analyses.

A different way to deal with the problem is to use the generalized density evolution equation, which is a consequence of the principle of preservation of probability [2], and leads to writing a linear partial differential equation for any quantity whose Pdf has to be determined. The coefficients of this equations at each instant are function of the state variables, and therefore they can be obtained from the (deterministic) solution of the dynamic system. The probability density evolution method has been implemented into the MADY code, which has already the routines for dynamic analysis of plane, three-dimensional, or beam and shell-based structures [4]. To our knowledge, this method has not been used in studying masonry structures, despite that uncertainties in the constitutive parameters and geometry have particular relevance.

In this paper, the potential of the application of the generalized density evolution method to masonry constructions is investigated. Firstly, different numerical method to solve the density evolution equation have been checked and the time and space step refinements needed have been identified with reference to a simple SDOF problem. Then, some preliminary numerical results have been obtained for a masonry tower represented as a simple beam model, applying a seismic action and accounting for the uncertainties of the Young's modulus. The response in terms of displacement at the top of the tower has been compared with the results obtained via the Monte Carlo method.

\section{PROBABILISTIC METHOD}

Let

$$
M \ddot{Y}+f(\dot{Y}, Y)=B(Y, t) \xi(t), \quad Y(0)=Y_{0}, \dot{Y}(0)=Y_{1}
$$

be the discretized equations of motion, where $t \in[0, T]$ is the time, $Y, \dot{Y}, \ddot{Y}$ are the displacement, velocity and acceleration n-dimensional vectors, respectively, $M$ is the mass matrix, $f$ is the internal force vector, $B$ is the input force influence matrix, $\xi$ is the external excitation vector and $Y_{0}$ and $\dot{Y}_{0}$ are the initial displacement and velocity vector, respectively. Equations (1) can be rewritten as

$$
\dot{X}=A(X, t)+\bar{B}(X, t) \xi(t), \quad X(0)=X_{0},
$$


where

$$
X=\left(\begin{array}{c}
\dot{Y} \\
Y
\end{array}\right)
$$

is the state vector, and

$$
A(X, t)=\left(\begin{array}{c}
-M^{-1} f(Y) \\
\dot{Y}
\end{array}\right), \quad \bar{B}(X, t)=\left(\begin{array}{c}
-M^{-1} B(Y, t) \\
0
\end{array}\right), \quad X_{0}=\left(\begin{array}{l}
Y_{1} \\
Y_{0}
\end{array}\right) .
$$

If randomness are present, coming from the properties of the system, it is assumed that there is a probability space $(\Omega, \mathcal{B}, \mathbb{P})$, where $\Omega \subset \mathbb{R}^{m}$ is the sample space, a collection of outcomes $\omega, \mathcal{B}$ is the Borel $\sigma$-algebra of $\Omega$ and $\mathbb{P}$ is the probability measure.

If the deterministic problem is well posed then, at each instant $t$, equations (2) have one and only one solution

$$
X_{t}=H(\theta, t)
$$

with $\theta=\Theta(\omega)$ for some smooth real or vector function $\Theta$, with probability density function $p_{\Theta}(\theta)$. (It is supposed that there are no randomness in the initial conditions and that $X_{0}$ has been fixed once and for all). As a consequence of the 'conservation of probability', which implies $\dot{\Theta}=0$, it holds

$$
\dot{X}_{t}=\frac{\partial H(\theta, t)}{\partial t}
$$

We are interested in determining the probability density function $p_{Z}(z, t)$ of some random variable $Z_{t}=\phi \circ X_{t}$, where $\phi: \mathbb{R}^{n} \rightarrow \mathbb{R}$ is a smooth and injective function. In order to determine function $p_{Z}(z, t)$, we proceed as proposed in [2]. Let $p_{Z \Theta}(z, \theta, t)$ be the joint probability density function of $Z_{t}$ and $\Theta$. Then $p_{Z \Theta}(z, \theta, t)$ is the solution of the linear PDE

$$
\frac{\partial p_{Z \Theta}(z, \theta, t)}{\partial t}+\dot{Z}_{t} \frac{\partial p_{Z \Theta}(z, \theta, t)}{\partial z}=0
$$

with the initial condition

$$
p_{Z \Theta}(z, \theta, 0)=\delta\left(z-z_{0}\right) p_{\Theta}(\theta)
$$

where $z_{0}=\phi\left(X_{0}\right)$. Once $p_{Z \Theta}(z, \theta, t)$ has been numerically determined by the finite difference method, the marginal probability density function $p_{Z}(z, t)$ can be obtained by the equation

$$
p_{Z}(z, t)=\int_{\Omega} p_{Z \Theta}(z, \theta, t) d \theta .
$$

\section{CHOICE OF THE DIFFERENCE SCHEME}

In order to choose a difference scheme and select the appropriate parameters for solving Eq. (3), various schemes proposed in literature have been tested.

Specifically, three finite difference schemes have been used, a one-sided, a two-sided (LaxWendroff) and a Total Variation Diminishing (TVD) scheme. The latter is a generalization of 
the other two, to which it can be traced back with an appropriate choice of parameters. The effectiveness of each scheme has been evaluated with reference to a simple problem for which the explicit solution is available [2][3]. Namely, the problem of an undamped SDOF system with a random frequency, under free oscillations with the initial displacement $x_{o}=0.1 \mathrm{~m}$, and null initial velocity.

In order to guarantee the convergence, the ratio between the step-time $\Delta t$ and the space mesh size $\Delta x$, denoted by $\lambda$, has been assumed equal to 0.1 . Some investigations have pointed out that, once the stability condition is guaranteed, the accuracy of the results does not significantly depend on the value of $\lambda$ as well as $\Delta t$. Conversely, the choice of $\Delta x$ as well as the number of the deterministic responses, equal to the number of samples, can appreciably affect the obtained results. In the following, the largest $\Delta x$ considered is equal to $3.125 * 10^{-3} \mathrm{~m}$, together with the corresponding $\Delta t=3.125 * 10^{-4} \mathrm{~m}$. Then, by mantaining the same value of $\lambda$, the equation has been solved by assuming a step in space equal to $\Delta x / 2$ and $\Delta x / 4$. In addition, the solution has been evaluated by considering both 25,50 and 100 deterministic responses (number of samples).

The obtained results are plotted in the following figures, and compared with the explicit solution. In particular, Figures 1, 2 and 3 show the mean value and standard deviation of $x$ as a function of $t$ obtained respectively with the three method. Figures 4 and 5 show the probability
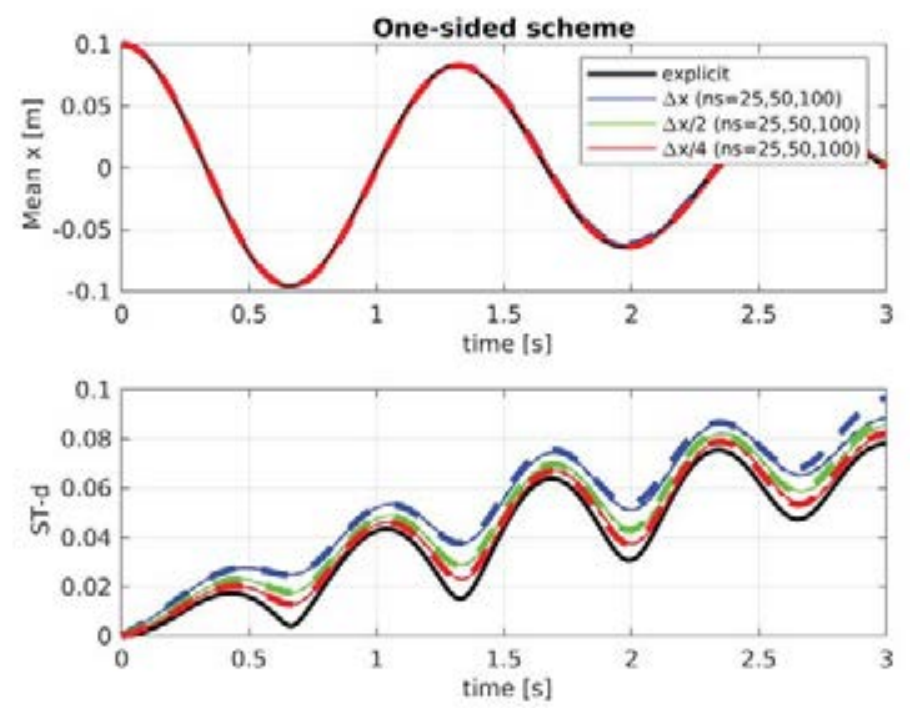

Figure 1: Mean value and standard deviation of $x$ vs $t$ given by the one-sided scheme.

density function, obtained with the three schemes considered at $t=0.9 \mathrm{~s}, t=1 \mathrm{~s}$ and $t=1.1 \mathrm{~s}$, for the spatial mesh size equal to $\Delta x, \Delta x / 2$ and $\Delta x / 4$, while Figure 6 shows the distribution function (Cdf) for $t=0.9 \mathrm{~s}$, for the considered different schemes and 100 samples.

Figures 1, 2, 3 evidence that the standard deviation is more sensitive than the mean value to the mesh size, whatever the scheme used. Moreover, it is evidenced that the best results are obtained with the TVD scheme.

This is also confirmed by the results shown in Figures 4 and 5. In particular, Figure 4 shows that the solution obtained with the scheme of Lax-Wendroff oscillates near the discontinuity, also assuming negative values.

Overall, these figures suggest that, for all the schemes used, the best results are obtained from 

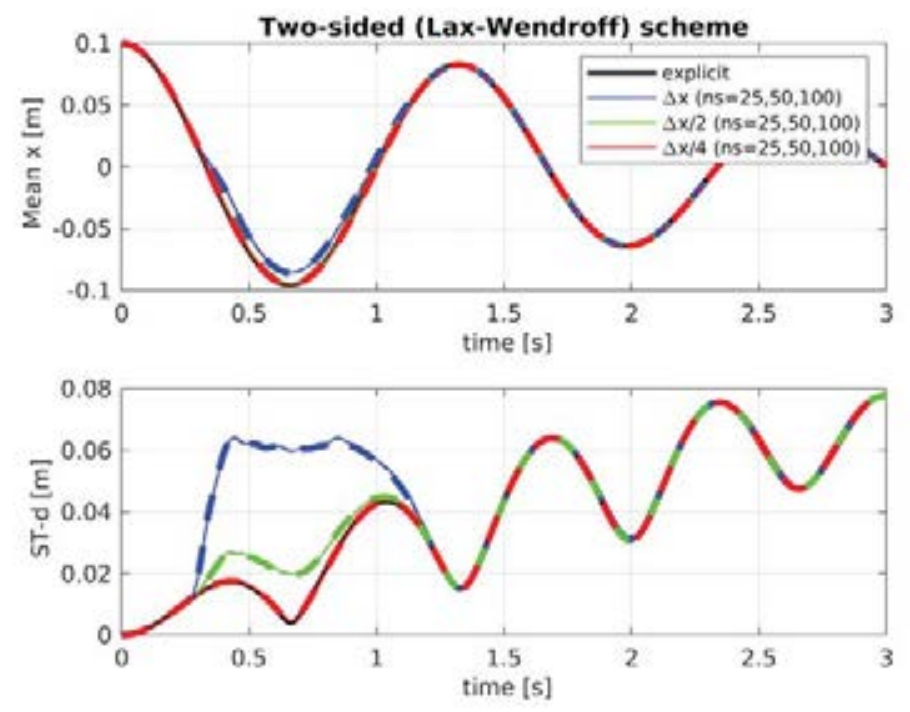

Figure 2: Mean value and standard deviation of $x$ vs $t$ given by the two-sided scheme.

an appropriate combination of the number of samples with the amplitude value of the mesh size. Lastly, it should be noted that the Cdf, shown in Figure 6, is little influenced by the choice of the calculation scheme.

\section{DETERMINISTIC MODEL}

The model used is a continuous beam model with a hollow rectangular cross-section and distributed mass $m$. It is implemented into the finite element code MADY and makes use of a constitutive equation formulated in terms of generalized stress and strain, i.e. $N=N(\epsilon, \kappa)$, $M=M(\epsilon, \kappa)$ [5], [6]. This constitutive law has been developed assuming that the sections remain plane, accounting for the axial stress alone $\sigma_{z}$, and describing the masonry behavior by means of a law $\sigma_{z}=\sigma_{z}\left(\epsilon_{z}\right)$. In particular, masonry is assumed to have a null tensile strength and a limited compressive strength $\sigma_{c}$. A softening behavior in compression is accounted for, and a linear piecewise law is defined as a function of the strain $\mu=\epsilon_{u} / \epsilon_{c}$ (Fig. 7). A damage function $\alpha$ has been defined as a function of the non-dimensionalized generalized strains $\eta=\epsilon / \epsilon_{c}$, $\chi=\kappa h / \epsilon_{c}$, such that the damaged beam section has reduced mechanical properties - Young's modulus $E$ and $\sigma_{c}$ - with respect to the undamaged one. For the sake of brevity it is not described in detail here, but an analogous procedure can be found in [7], [8], [9] for other types of sections.

The actual geometry of the Torre Grossa of S. Gimignano used as reference real tower has been approximated by the beam model with a rectangular cross-section and an overall height of $50 \mathrm{~m}$ [10]. The constraint offered by the neighbouring buildings extending for a height of $20 \mathrm{~m}$ from the soil has been modelled with a set of lateral elastic links (Figure 7).

\section{RESULTS}

The PDEM has been applied to the study of the tower modelled as shown in Fig. 7. The deterministic nonlinear dynamic analyses have been conducted by applying an input ground motion recorded during the Tabas, Iran event of 1978; the accelerogram has a magnitude of 7.4, a duration of $63.40 \mathrm{~s}$ and a PGA of $0.925 \mathrm{~g}$.

The main pourpose of the study presented here is to verify the effectiveness of the method for such dynamic systems which, differently from the simple oscillator studied above, have strong 

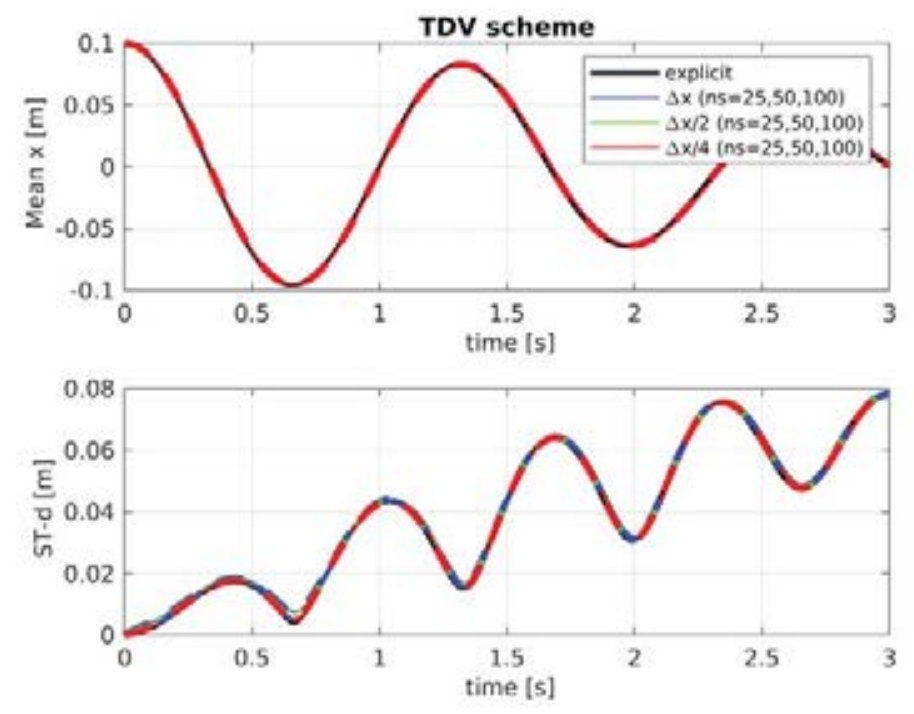

Figure 3: Mean value and standard deviation of $x$ vs $t$ given by the TDV scheme.
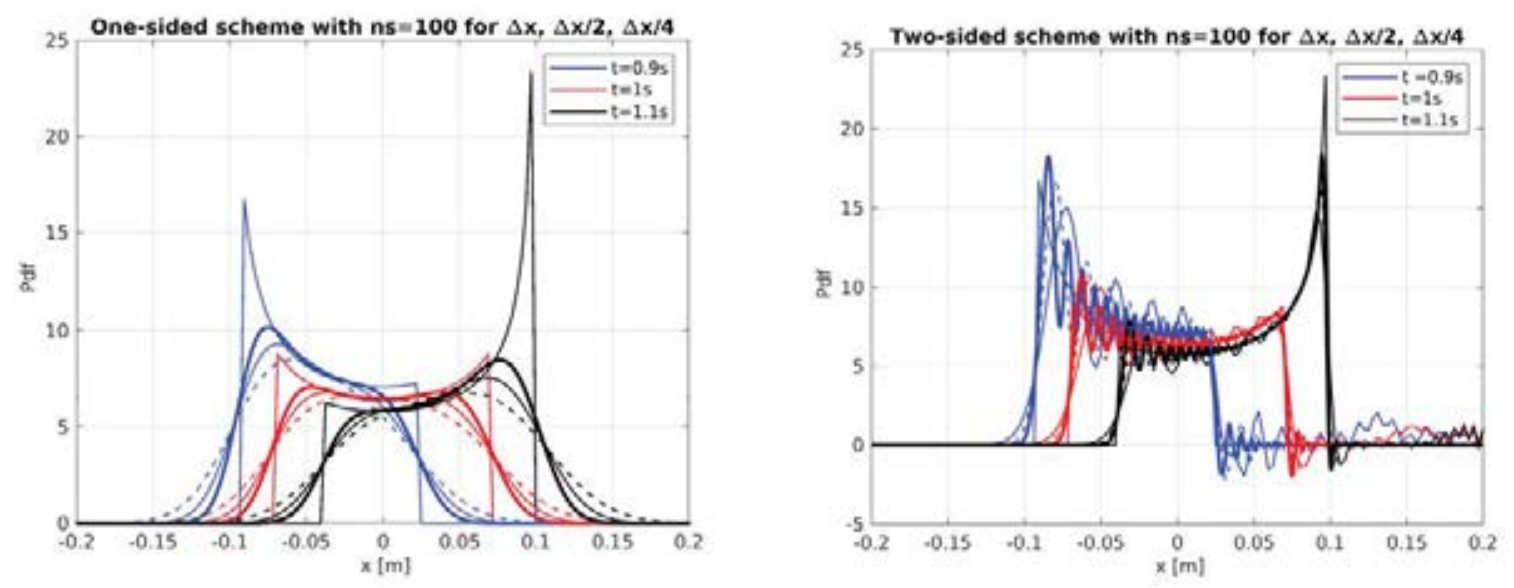

Figure 4: Pdf obtained via the one-sided and two-sided scheme.

non-linearities. On the basis of the results described in the foregoing, the TDV scheme has been used for the integration of equation (3). Moreover, the investigation is limited to determining the trend over time of the Pdf of the displacement at the top of the tower, assigned the Pdf of the Young's modulus of the material, a mechanical parameter that greatly affects the response of masonry structures. For this parameter, a mean value $\mu=1.8 \mathrm{GPa}$ and a uniform distribution on the interval [1.195, 2.405] have been assumed. The results obtained with PDEM at some timesteps, for various values of both the number of samples (ns) and the mesh size are compared with those provided by the Monte Carlo Method (MC), with 50,000 samples.

Figure 8 shows the mean and standard deviation as function of time, while Figure 9 shows the Pdf at four steps of time, calculated with the PDEM. Once again, it is observed that the best results are obtained with an appropriate combination of the number of samples and the length of the mesh size. Figure 10 shows the comparison of the Pdfs, obtained with $\Delta x / 4$ and ns $=200$, with those deduced via MC for ns $=50000$. In all cases, it is evidenced that the Pdf calculated with PDEM is nonzero in a wider range than that obtained with MC. Nevertheless, 

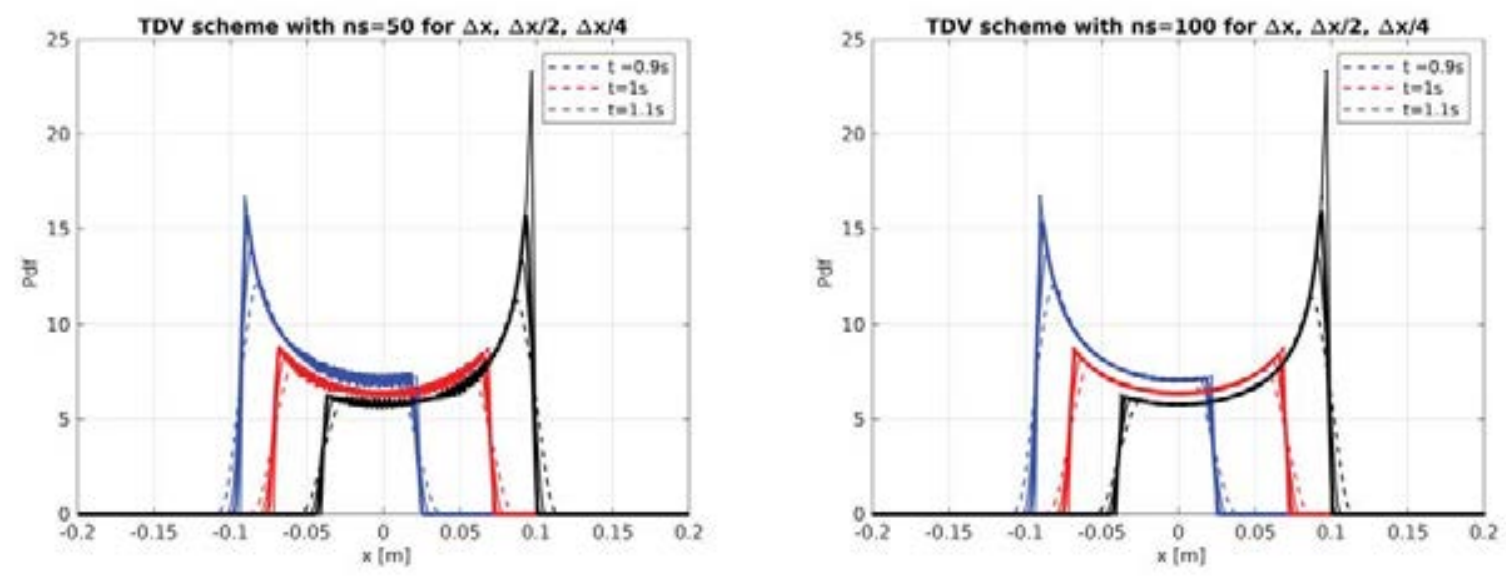

Figure 5: Pdf obtained via the TDV scheme by varying the number of samples ns and $\Delta x$.

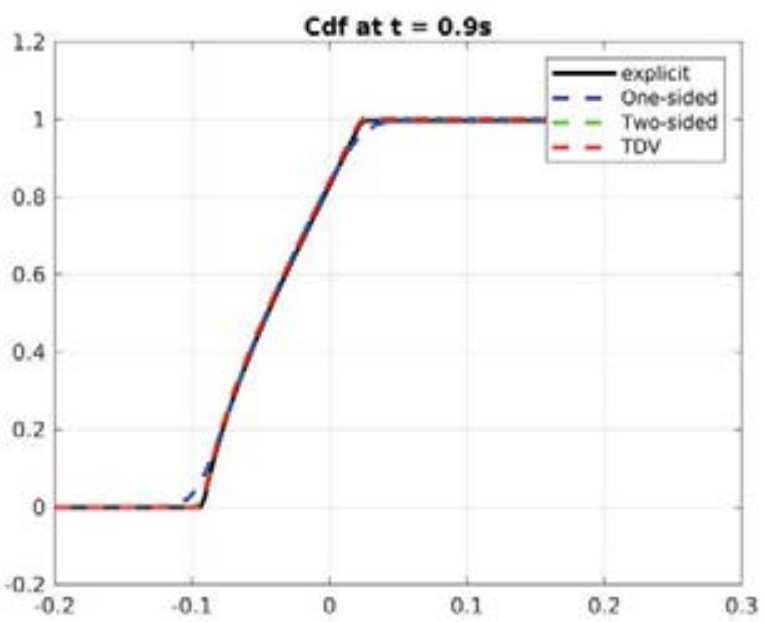

Figure 6: Cdf obtained via the different schemes compared to the explicit one.

the comparison between the Cdf shown in Figure 11, evidences a good agreement between the results obtained with the two different methods.

\section{CONCLUSIONS}

In the paper, the probabilistic density evolution method is proposed for analysing the seismic response of historic masonry structures. Developed at the earlies 2000, the method is applied here for the first time to the study of masonry towers.

Some comparisons with the Monte Carlo method shows the accuracy of the results obtained by the PDEM, that is much less consuming than the former.

Given the high incertainties involved in the analyses of complex masonry structures, their high non-linearities, the large size and articulated geometries, the method appears particularly suitable for the assessment of the dinamic response of such structures, given the low number of deterministic results needed for a good prevision of the incertainties propagation. 

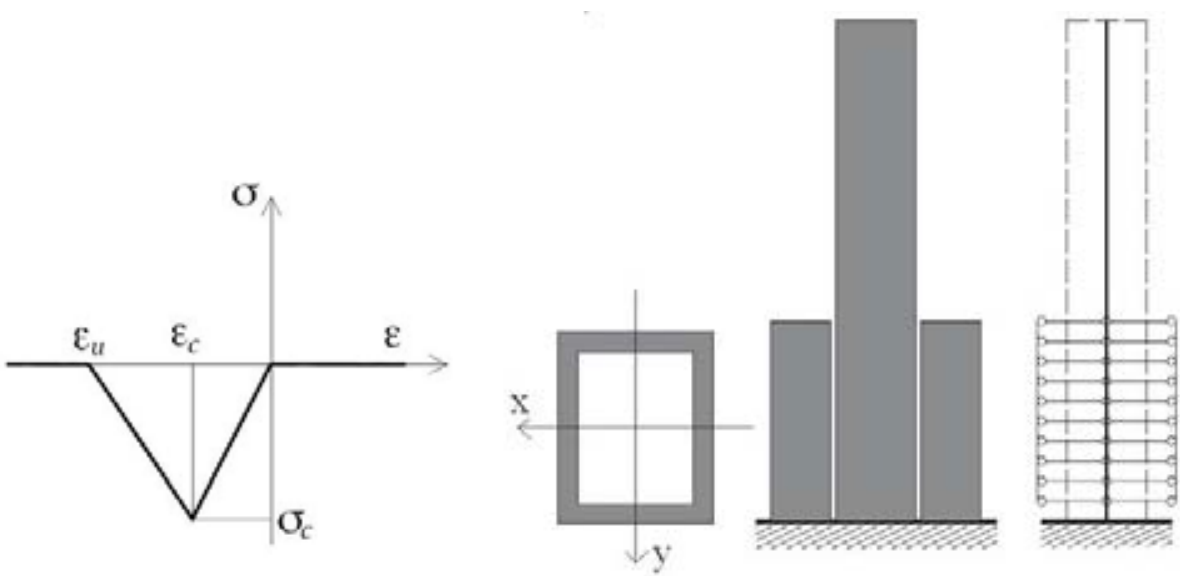

Figure 7: (from the left) Masonry behaviour, simplified geometrical scheme of the tower, and FE mesh.

\section{REFERENCES}

[1] E. Zio, The Monte Carlo simulation method for system reliability and risk analysis, Springer-Verlag, 2013.

[2] J. Li, J. Chen, Stochastic dynamics of structures, J. Wiley and Sons, 2009.

[3] J. Li, J. B. Chen, Probability density evolution method for dynamic response analysis of structures with uncertain parameters, Computational Mechanics, 34, 400-409, 2004.

[4] M. Lucchesi, B. Pintucchi, N. Zani, MADY, a computer code for numerical modelling masonry structures. in preparation, 2020.

[5] M. Lucchesi, B. Pintucchi, A numerical model for non-linear dynamics analysis of masonry slender structures. European Journal of Mechanics A/Solids, 26, 88-105, 2007.

[6] M. Lucchesi, B. Pintucchi, M. Šilhavý, N. Zani, On the dynamics of viscous masonry beams. Continuum Mechanics and Thermodynamic, 27, 349-365, 2015.

[7] M. Lucchesi, B. Pintucchi, N. Zani, Dynamic analysis of FRP-reinforced masonry arches via a no-tension model with damage. A. Di Tommaso, C. Gentilini, and G. Castellazzi eds. Key Engineering Materials, 624 KEM,619-626, 2015.

[8] B. Pintucchi, N. Zani, A simple model for performing nonlinear static and dynamic analyses of unreinforced and FRP-strengthened masonry arches. European Journal of Mechanics /A Solids, 59, 210-231, 2016.

[9] B. Pintucchi, Dynamic analysis of FRP-strengthened masonry. B. Ghiassi, G. Milani eds. Numerical Modeling of Masonry and Historical Structures: From Theory to Application, 659-681, 2019.

[10] G. Bartoli, M. Betti, S. Giordano, In situ static and dynamic investigations on the Torre Grossa masonry tower Engineering Structures, 52, 718-733, 2013.

[11] M. Lucchesi, B. Pintucchi, N. Zani, The generalized density evolution equation for the dynamic analysis of slender masonry structures. A. Di Tommaso, C. Gentilini, and G. Castellazzi eds. Key Engineering Materials, 817 KEM, 350-355, 2019. 

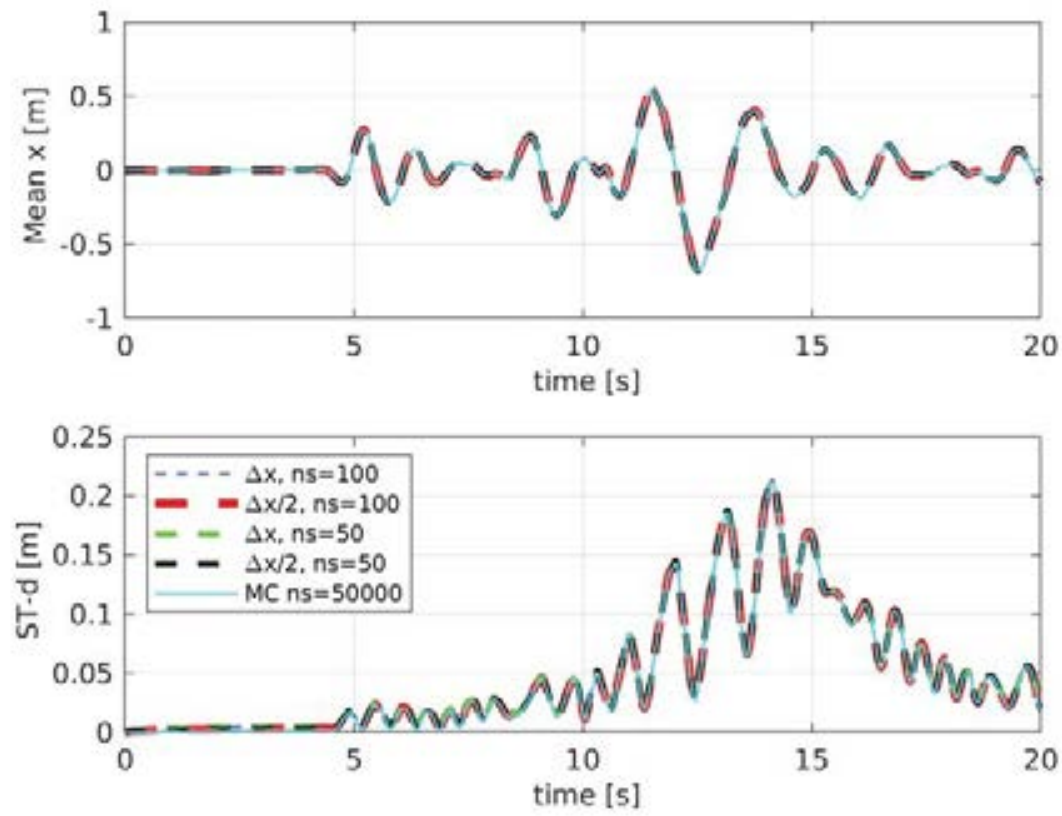

Figure 8: Mean and standard deviation obtained with the PDEM compared to the MC results.
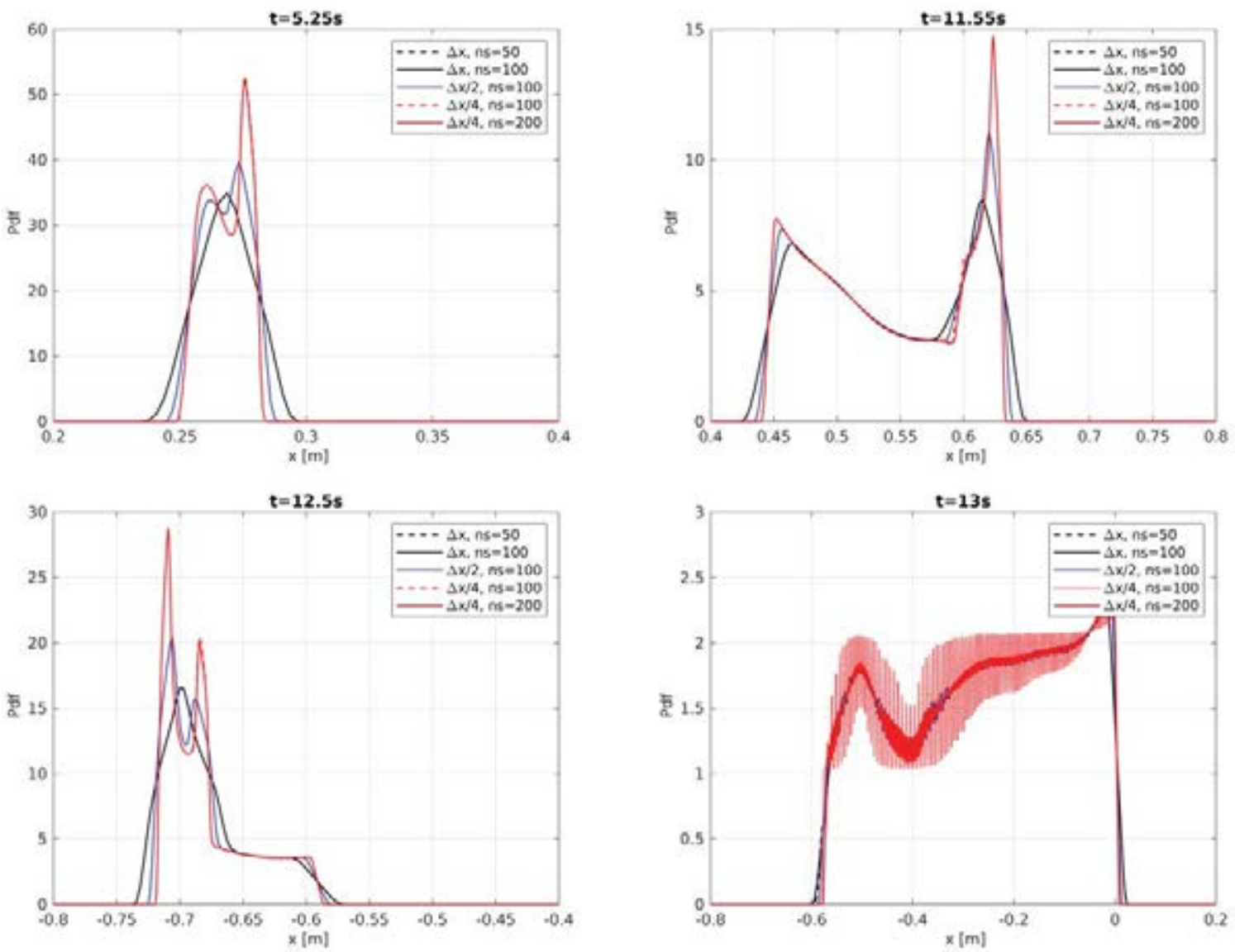

Figure 9: Pdf obtained by varying $\Delta x$ and the number of samples (ns). 

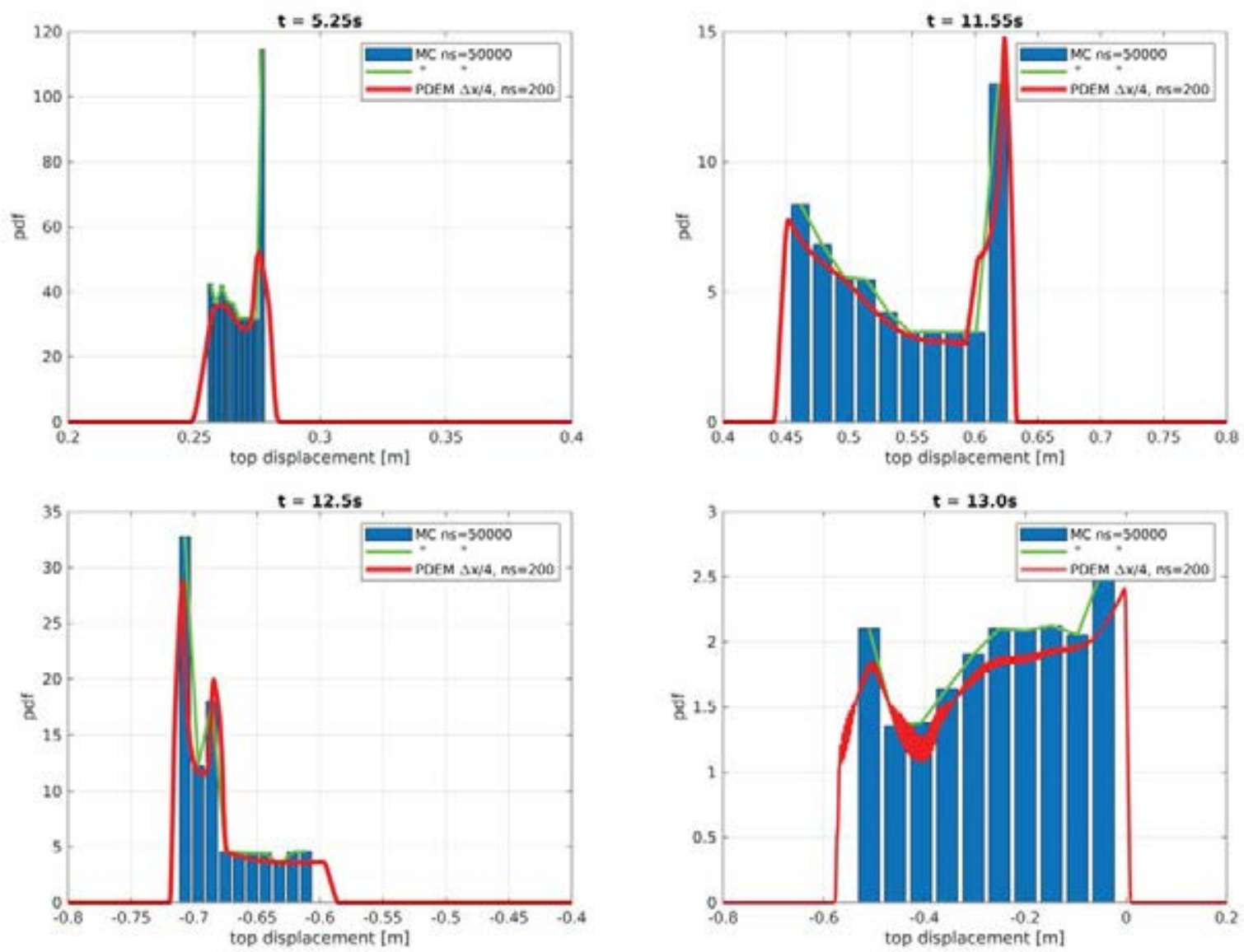

Figure 10: Pdf obtained via the PDEM compared with the MC results.

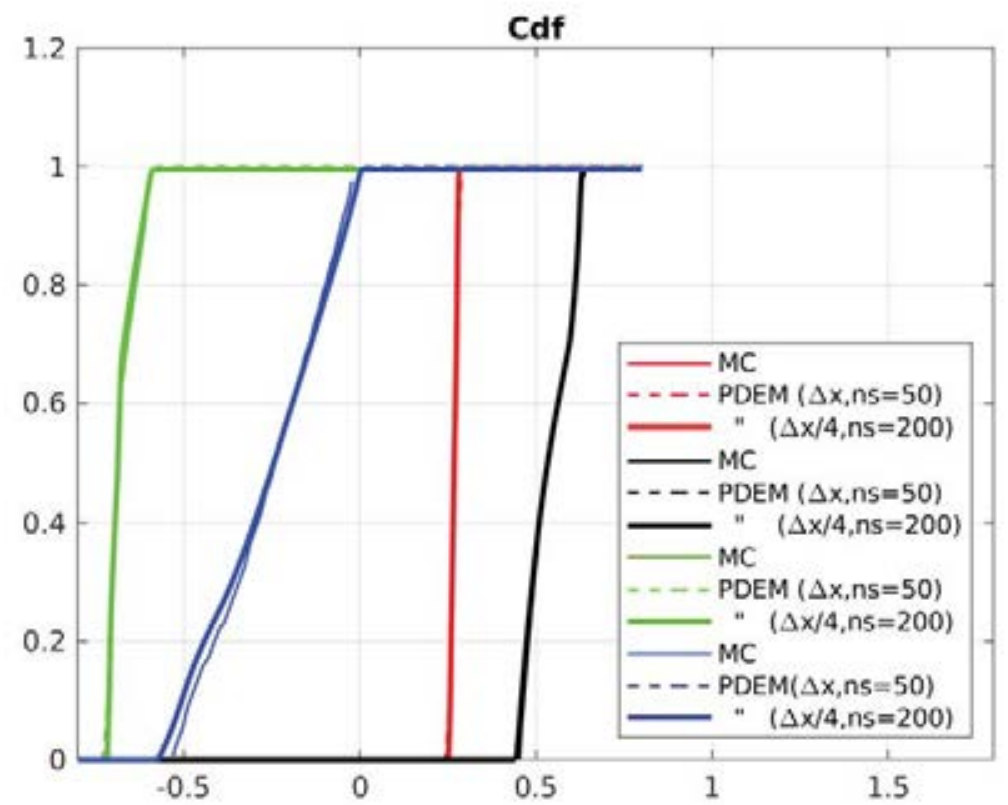

Figure 11: Cdf obtained via the PDEM compared with those from MC results (red lines for $t=5.25 \mathrm{~s}$, black for $t$ $=11.55 \mathrm{~s}$, green for $t=12.5 \mathrm{~s}$ and blue for $t=13 \mathrm{~s}$ ). 